\title{
Verträge im freien Markt
}

\section{Die Auswirkungen des Persönlichen Budgets auf die Verein- barungen zwischen Budgetnehmern und Leistungserbringern}

Stefan Schick

Prof. Dr. Stefan Schick ist als

Rechtsanwalt und Fachanwalt für Steuerrecht in Stuttgart tätig. Zudem lehrt er an der European Business School in Oestrich-Winkel im Intensivstudium Gesundheitsökonomie und an der SRH Fachhochschule für Gesundheit Gera. E-Mail Stefan.Schick@reithschick.de
Beim Persönlichen Budget muss der Vertragsgestaltung zwischen Leistungserbringer und Leistungsberechtigtem auch unter Verbraucherschutzgesichtspunkten besondere Aufmerksamkeit gewidmet werden.

Während im sozialrechtlichen Leistungsdreieck die Leistungsbeziehungen zwischen den Leistungserbringern und den Kostenträgern sozialrechtlich geregelt werden, fehlt diese Rechtsbeziehung beim Persönlichen Budget. Mehr noch: An die Stelle der sozialrechtlichen treten zivilrechtliche Vereinbarungen zwischen Budgetnehmern und Leistungserbringern, für die andere Grundsätze gelten. Der vorliegende Beitrag beschäftigt sich mit den Auswirkungen auf die vertraglichen Beziehungen zwischen Leistungserbringern und Leistungsempfängern.

Nachfolgend werden zunächst die wesentlichen Vertragsinhalte zusammengefasst (vgl. 1.). Da jeder Leistungserbringer eine Vielzahl von - gleich formulierten Verträgen abschließt, gelten für sie die gesetzlichen Regelungen für Allgemeine Geschäftsbedingungen (AGB, $\mathbb{S} S 305 \mathrm{ff}$. $\mathrm{BGB})$. Wesentliche Aspekte im $\mathrm{Zu}-$ sammenhang mit der bei AGB durchzuführenden gerichtlichen Inhaltskontrolle müssen daher beachtet werden (vgl. 2.).

Unabhängig von Vertragsinhalten und Inhaltskontrolle muss der Leistungserbringer darauf achten, dass der Vertrag wirksam zustande kommt. Bestehen Zweifel an der Geschäftsfähigkeit des Leistungsempfängers als Vertragspartner, so ist die Bestellung eines Betreuers $(\mathbb{S} \mathbb{S}$ 1896 ff. BGB) anzustreben.

\section{Wesentliche Inhalte der zwischen Leistungserbringern und Budgetnehmern abzuschließenden Verträge}

Budgetnehmer und Leistungserbringer schließen zivilrechtliche Verträge $a b$, für die insbesondere die Bestimmungen des Bürgerlichen Gesetzbuchs (BGB) gelten. Handelt es sich beim Leistungserbringer um eine Pflegeeinrichtung im Sinne des SGB XI, so gelten zwar die Versorgungsverträge $(\mathbb{S} 73$ SGB XI) und die Rahmenverträge ( $\mathbb{S} 75$ SGB XI) nicht unmittelbar für die Leistungsbeziehung zwischen Leistungserbringern und Budgetnehmern. Diese können aber durch Verweisung in den Vertrag einbezogen werden. Dies setzt voraus, dass sie dem Budgetnehmer bekanntgegeben, also am Besten dem Vertrag beigefügt werden. Da jede Pflegeeinrichtung nach $\mathbb{S} \mathbb{S} 112$ ff. SGB XI bestimmte Qualitätsanforderungen erfüllen muss, gelten diese Voraussetzungen zumindest im Rahmen einer Einbeziehung in den Vertrag auch für Leistungen im Rahmen eines Persönlichen Budgets.

Ausgangspunkt für den Vertrag ist die Zielvereinbarung, die Leistungsträger und Budgetnehmer abschließen. Sie ist eine sozialrechtliche, also öffentlichrechtliche Vereinbarung, mit der sich der Budgetnehmer verpflichtet, das ihm gewährte Budget zur Erreichung der vereinbarten Ziele einzusetzen. Die Zielvereinbarung wirkt damit in die zwischen dem Budgetnehmer und dem Leistungserbringer abzuschließenden Vereinbarungen hinein und gibt deren Anforderungen vor. Für die Grundstruktur der Vereinbarungen zwischen Leistungserbringern und Budgetnehmern sind zwei Konstellationen denkbar:

- Der Budgetnehmer kauft alle Leistungen (Grundleistungen, die pauschal vergütet werden; Einzelleistungen, die optional in Anspruch genommen werden können) bei einem Leistungserbringer ein.

- Der Budgetnehmer kauft Leistungen bei unterschiedlichen Leistungserbringern ein. Dann stellt sich die Frage, wie die Gesamtqualität bei einer Leistungserbringung durch verschiedene Leistungserbringer gesichert werden kann. 
Im Einzelnen sind insbesondere Vereinbarungen zu treffen über

- Art und Zeitpunkt der Erbringung von Grundleistungen, die pauschal vergütet werden

- Art und Zeitpunkt der Erbringung von Einzel- und Wahlleistungen, die optional in Anspruch genommen und gesondert berechnet werden

- die Vergütung. Dabei sind denkbar:

- Pauschalvergütungen für bestimmte, vereinbarte Leistungen. Da im Rahmen der Pauschalvergütung erbrachte Leistungen nicht gesondert berechnet werden können, muss die Leistungspflicht so konkret festgelegt werden, dass es keine Meinungsverschiedenheiten über die pauschal vergüteten Leistungen gibt und die Leistung sicher kalkulierbar ist.

- Einzelvergütungen vor allem für optionale Leistungen. Standardleistungen können nach einem »Gebührenkatalog « oder nach Zeitaufwand abgerechnet werden.
- die Geheimhaltung aller nicht allgemein bekannten Umstände über den anderen Partner. Dies gilt insbesondere für Gesundheitszustand und Hilfebedarf des Budgetnehmers.

- generelle Sorgfaltspflichten beider Vertragspartner

- die Laufzeit und Kündigung des Vertrags. Dabei sind die ordentliche Kündigung und die außerordentliche (fristlose) Kündigung aus wichtigem Grund zu unterscheiden. Für die AGB-rechtliche Angemessenheit sind zunächst die gesetzlichen Bestimmungen als "Wertungsmaßstab « von Bedeutung. Da es sich bei den Vereinbarungen zwischen Leistungserbringern und Budgetnehmern i.d.R. um Dienstverträge im Sinne des BGB handelt, gilt mangels abweichender Vereinbarungen Folgendes:

- Ordentliche Kündigungen: bei einer Vergütung der Leistung nach Tagen kann an jedem Tag für den Ablauf des Folgetages, bei einer Vergütung

\section{»Gegen Allgemeine Geschäftsbedingungen können auch Verbraucherschutzorganisationen gerichtlich vorgehen"}

- die Fälligkeit der Vergütung. Dabei kommt für die Abrechnung von Pauschalvergütungen eine zeitraumbezogene Abrechnung in Betracht. Bei Einzelleistungen ist die Abrechnung unmittelbar nach Leistungserbringung, aber auch zeitraumbezogen denkbar. Welcher Abrechnungsmodus gewählt wird, ist vom Leistungsumfang abhängig.

- Regelungen über die Anpassung der Vergütung. Entsprechende Klauseln müssen klar nachvollziehbar sein und den AGB-rechtlichen Anforderungen Stand halten (vgl. dazu unten 2.).

- die vertrauensvolle Zusammenarbeit, vor allem über die Mitwirkung des Budgetnehmers zur Erreichung der in der Zielvereinbarung vereinbarten Ziele, aber auch gegenseitige Informationspflichten. Diese betreffen vor allem medizinisch indizierte Veränderungen des Hilfebedarfs des Budgetnehmers. der Leistungen nach Wochen spätestens am ersten Werktag einer Woche für den Ablauf des folgenden Sonnabends und bei einer Vergütung nach Monaten spätestens am 15. des Monats auf das Ende des Kalendermonats gekündigt werden.

- Als Gründe für die außerordentliche (fristlose) Kündigung aus wichtigem Grund kommen in Betracht:

- Die vereinbarte Betreuung kann vor allem aufgrund einer Verschlechterung des Zustandes des Budgetnehmers - nicht mehr gewährleistet werden.

- Der Budgetnehmer benötigt die Leistung nicht mehr.

- Der Budgetnehmer verstirbt.

- die Folgen der Kündigung. Die Leistungserbringer sind bei ordentlichen Kündigungen an möglichst langen Kündigungsfristen und bei fristlosen
Kündigungen an einer längeren Fortzahlung der Vergütung durch den Budgetnehmer interessiert. Dem steht das Interesse des Budgetnehmers gegenüber, den Vertrag kurzfristig beenden zu können, ohne dass ihn Zahlungspflichten treffen. Insoweit muss ein angemessener Interessenausgleich gesucht werden. Die Angemessenheit kann nach den AGB-rechtlichen Grundsätzen gerichtlich überprüft werden (vgl. dazu nachfolgend 2.).

\section{Auswirkungen des Rechts der AGB auf die vertraglichen Beziehungen im Rahmen des Persönlichen Budgets}

Im Rahmen des Persönlichen Budgets werden in einer Vielzahl von Fällen vergleichbare vertragliche Regelungen getroffen. Dabei kommen formlose, mündliche Vereinbarungen nur in einfachen Ausnahmefällen in Betracht. Es werden daher vorformulierte Vertragsregelungen verwendet, auf die das Recht der AGB $(\mathbb{S} 305 \mathrm{ff}$. BGB) Anwendung findet. Auch Verhandlungen über vertragliche Einzelregelungen sind selten. Damit sind beim Vertragsabschluss die folgenden Aspekte zu beachten:

- Die vorformulierten Vertragsbedingungen müssen ausdrücklich in den Vertrag einbezogen werden $(\mathbb{S} \mathbb{S} 305,305$ a BGB). Soweit es sich um Musterverträge handelt, die unterzeichnet werden, dürfte diese Anforderung ohne weiteres erfüllt sein. Problematisch ist dies allerdings dann, wenn andere Vereinbarungen oder Unterlagen einbezogen werden sollen.

- Individualabreden haben Vorrang $(\mathbb{S}$ 305 b BGB). Diese müssen klar und deutlich dokumentiert werden, damit es später keine Auseinandersetzungen beispielsweise über einen abweichenden Leistungsumfang oder eine abweichend vereinbarte Vergütung gibt.

- überraschende Klauseln sind unzulässig (\$ 305 c Abs. 1 BGB)

- Unklarheiten gehen zulasten des Verwenders der AGB ( $\$ 305$ c Abs. 2 BGB)

- Die vereinbarten Regelungen dürfen nicht unangemessen sein. AGB unterliegen gegebenenfalls einer gerichtlichen Inhaltskontrolle. Diese kann nicht nur durch die Budgetnehmer als Vertragspartner, sondern auch durch 
Verbraucherschutzorganisationen, wie die Verbraucherzentralen, veranlasst werden. Im Rahmen der Inhaltskontrolle werden für die Angemessenheit einer vertraglichen Regelung die gesetzlichen Bestimmungen herangezogen (vgl. zur Kündigungsregelung insbesondere die Ausführungen oben 1.).

Für die Inhaltskontrolle gelten die $\mathbb{S} \mathbb{S}$ 307 ff. BGB. Danach sind Bestimmungen in AGB unwirksam, wenn sie den Budgetnehmer unter Verstoß gegen Treu und Glauben unangemessen benachteiligen. Darunter fällt auch der Fall, dass die Bestimmung nicht klar und verständlich ist. Nach $\mathbb{3} 307$ Abs. 2 BGB ist eine unangemessene Benachteiligung im Zweifel anzunehmen, wenn eine Bestimmung

- mit wesentlichen Grundgedanken der gesetzlichen Regelung, von der abgewichen wird, nicht zu vereinbaren ist oder

- wesentliche Rechte und Pflichten, die sich aus der Natur des Vertrags ergeben, so einschränkt, dass die Erreichung des Vertragszwecks gefährdet ist.

Neben diesen allgemeinen Grundsätzen für die Inhaltskontrolle wird in $\mathbb{S} \mathbb{S} 308$ und 309 BGB unterschieden in sogenannte Klauselverbote mit Wertungsmöglichkeit $(\mathbb{S} 308$ BGB) und in sogenannte Klauselverbote ohne Wertungsmöglichkeit ( $\mathbb{S} 309$ BGB). Enthalten die AGB oder Musterverträge Klauseln, die von $\mathbb{S}$ 308 BGB erfasst werden, so kann sie das Gericht als wirksam oder als unwirksam ansehen. Bei Regelungen, die von $\mathbb{} 309$ BGB erfasst werden, besteht diese Wertungsmöglichkeit nicht, solche Regelungen sind also in jedem Falle unwirksam.

Nachfolgend werden die wesentlichsten Einzelregelungen ohne Anspruch auf Vollständigkeit kurz zusammengefasst. Wichtige Klauseln mit Wertungsmöglichkeit sind insbesondere

- unangemessen lange Fristen für die Abgabe von Erklärungen oder Erbringung von Leistungen

- unangemessene Änderungsvorbehalte zugunsten des Leistungserbringers. Dies betrifft beispielsweise die Anpassung von Vergütungen aus Dauerschuldverhältnissen. Dann müssen entweder die Voraussetzungen für die Anpassung der Vergütung konkret umschrieben oder - falls die Anpassungsmöglichkeit über eine Steigerung in Höhe des Lebenshaltungskostenindexes hinausgeht - ein außerordentli- ches Kündigungsrecht des Leistungsberechtigten vorgesehen werden.

- die unangemessene Vergütung von Aufwendungen des Leistungserbringers bei Kündigungen. Ausdrückliche gesetzliche Bestimmungen für zulässige Regelungen bestehen nicht. Die Obergrenze für die Fortzahlungspflicht des Budgetnehmers dürfte bei vier bis höchstens acht Wochen liegen. Gegebenenfalls muss sich der Leistungserbringer ersparte Aufwendungen anrechnen lassen.

Wichtige Klauseln ohne Wertungsmöglichkeit sind insbesondere

- der Ausschluss des Leistungsverweigerungsrechts oder der Aufrechnung des Leistungsberechtigten mit einer unbestrittenen oder rechtskräftig festgestellten Forderung

- die unangemessene Pauschalierung von Schadensersatzansprüchen

- der Haftungsausschluss bei Verletzung von Leben, Körper, Gesundheit und bei grobem Verschulden.

\section{Fazit}

Die Leistungserbringer müssen sich nicht nur im Hinblick auf die Leistungen selbst, sondern auch im Zusammenhang mit vorformulierten Vertragsbedingungen dem Markt stellen. Für die Inhalte der Vereinbarungen mit den Budgetnehmern bilden die mit den Leistungsträgern abgeschlossenen Regelungen - gewissermaßen als Check-Liste - einen ersten Anhaltspunkt. Da die von Leistungserbringern und Budgetnehmern abzuschließenden Vereinbarungen in der Regel in vielen Fällen verwendet werden, müssen sie die Anforderungen des Rechts der Allgemeinen Geschäftsbedingungen ( $\mathbb{S} 305$ ff. BGB) erfüllen.

\section{Das Berater- handbuch zum Ausländerrecht}

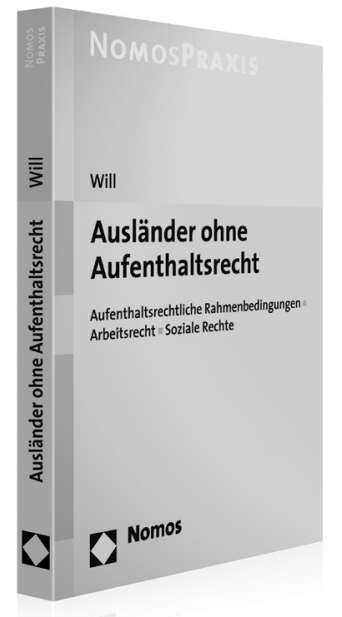

\section{Ausländer ohne Aufenthaltsrecht}

Aufenthaltsrechtliche Rahmenbedingungen | Arbeitsrecht | Soziale Rechte

Von RAin Prof. Dr. Annegret Will

2008, 296 S., brosch., 39,-€, ISBN 978-3-8329-2500-O

Für die Beratung von Ausländern, die kein Aufenthaltsrecht (mehr) oder nur ein prekäres Aufenthaltsrecht besitzen sind Kenntnisse des Aufenthalts-, Arbeits- und Sozialrechts ebenso unentbehrlich wie straf- und datenschutzrechtliches Wissen.

Das Handbuch behandelt sämtliche Rechtsfragen, die sich in der Beratungsarbeit stellen. Die systematische Einbettung der Darstellung in die jeweiligen Rechtsgebiete gewährleistet den für eine rasche Einarbeitung unentbehrlichen Überblick über die einzelnen Rechtsmaterien. Die Probleme werden anhand von Beispielen leicht verständlich und praxisnah erläutert.

\section{Nomos}

Bitte bestellen Sie im Buchhandel ode versandkostenfrei unter $\$ www.nomos-shop.de 
"Der Kunde kann sich jede Farbe wünschen, vorausgesetzt, die Farbe ist Schwarz."

Henry Ford, amerikanischer Industrieller (1863-1947)

"Wer eine Ware verkaufen will, muss den Markt studieren." Friedrich Dürrenmatt, Schweizer Schriftsteller (1921-1990)

"Ein weiser Verbrauch ist viel schwieriger als eine weise Herstellung. Was fünf Menschen herstellen, verbraucht ein einzelner leicht: Daher stellt sich für jeden einzelnen und für jedes Land nicht die Frage, wie man herstellen soll, sondern wie die Waren verbraucht werden sollen." John Ruskin, englischer Schriftsteller (1819-1900)

"Der Mensch entwickelt viel Unnatur, wo er seinesgleichen als Kunden behandelt, " Martin Kessel, deutscher Schriftsteller (1901-1990)

„Im Non-Profit-Bereich werden die materiellen

Geschäftsergebnisse stärker von politischen Entscheidungen als von der Zufriedenheit einzelner Kundengruppen beeinflusst. " Marianne Meinhold, deutsche Psychologin (geb. 1941)

"Kaufe nie etwas, was du eigentlich nicht willst, nur weil es billig ist; es wird dich teuer zu stehen kommen." Thomas Jefferson (1743-1826), amerikanischer Politiker, Verfasser der amerikanischer Unabhängigkeitserklärung und 3. Präsident der USA

"Jeder bedarf des gemeinsamen Schutzes, der gemeinsamen Einrichtungen, die er nicht geschaffen hat. Das Dach, unter dem er schläft, die Straße, die er betritt, das Werkzeug, das er hebt, dies alles ist von der Gesamtheit geschaffen, und er hat nur den Teil daran, den Übereinkommen und Herkommen ihm zuweisen. Wirtschaft ist nicht Privatsache." Walter Rathenau, deutscher Industrieller und Politiker (1867-1922) 